\title{
High performance metal-only fan-beam reflectarray with a delta source applicable for an electromagnetic fence
}

\author{
Yong-Heui Cho \\ School of Information and Communication Engineering \\ Mokwon University, Daejeon, 302-729, Republic of Korea
}

\begin{abstract}
The scattering solutions for multiple rectangular metallic gratings in a perfectly conducting plane excited by the TE and TM delta sources are presented using an overlapping T-block method. A reflectarray composed of rectangular metallic gratings shows fanbeam radiation patterns that are useful for an electromagnetic fence. The scattering characteristics of multiple rectangular gratings were computed in terms of total radiated power and antenna directivity. The design method of a fan-beam reflectarray to obtain high directivity was also compared with superdirective radiation and parabolic reflector phase.
\end{abstract}

Keywords: Electromagnetic Fence, Millimeter-Wave, Metal-Only Reflectarray, High-Gain Antenna.

\section{INTRODUCTION}

An electromagnetic fence [1]-[5] is a virtual fence operated by an electromagnetic wave that is unrecognizable with human eyes. Since the electromagnetic fence is physically non-existent, conceptual, and software-driven, human beings or animals can physically trespass over the fence. However, the software can perceive the trespassing and an alarm of intrusion may be transmitted to a central control unit with wireless network [6], [7]. This means that we can detect the locations of intruders very easily with divided and named electromagnetic fences. In order to increase the resolution of intrusion position, we need to increase operating frequency which results in smaller wavelength. Recently, a millimeter-wave electromagnetic fence has been investigated in [5], [8]. The millimeter-wave technology has been extensively studied to build up broadband multimedia wireless communication systems [9]. In [8], the millimeter-wave was used to detect foreign object debris on runways.

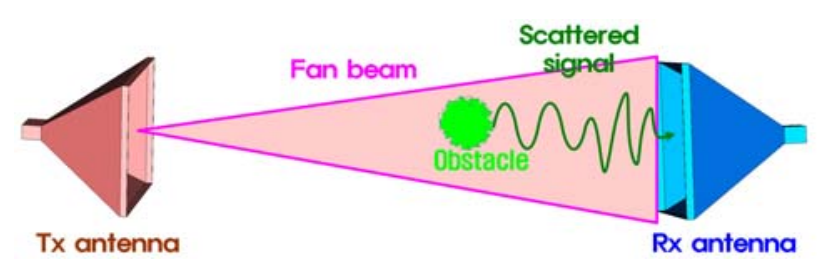

Fig. 1. Concept of electromagnetic fence

Fig. 1 shows a conceptual diagram of an electromagnetic fence in terms of bistatic operation. A transmitting (Tx) antenna in Fig. 1 radiates an electromagnetic wave to a

${ }^{*}$ Corresponding author. E-mail : yhcho@mokwon.ac.kr
Manuscript received Nov 14,2011 ; accepted Dec.05, 2011 receiving $(\mathrm{Rx})$ antenna. When obstacles are present in the line of sight (LOS) of an electromagnetic wave, they generate a scattered signal to an $\mathrm{Rx}$ antenna. At the $\mathrm{Rx}$ part, we can analyze received signals to determine the existence of obstacles and identify the signal signature of them. As is well known, the millimeter-wave has strong LOS characteristics [9] and the millimeter-wave frequency band facilitates precise operation of the electromagnetic fence. Among the components of a millimeter-wave communication system, it is not easy to increase output power of a high power amplifier (HPA). The cost of high performance HPA increases very rapidly with respect to output power. Therefore, we need to have very high gain antenna to satisfy the link budge of an electromagnetic fence system.

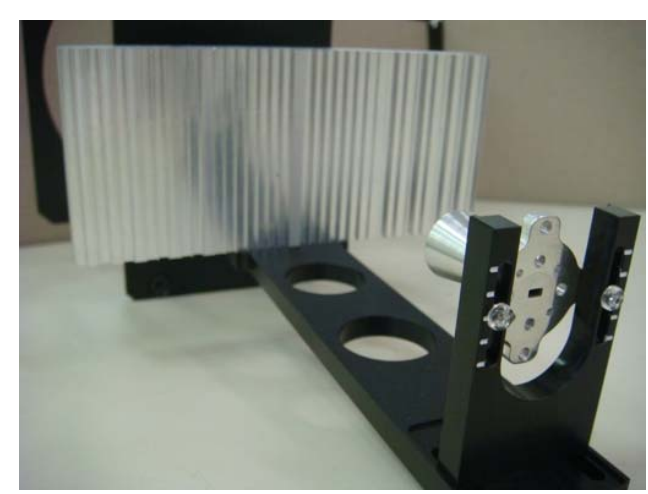

Fig. 2. Example of a two-dimensional metal-only reflectarray [13]

A reflectarray [10]-[13] was proposed in 1963 to obtain versatile beamforming characteristics. Since a reflectarray can be manufactured with metal only [13], a metal-only reflectarray shown in Fig. 2 is a promising candidate for millimeter-wave 
applications. A metal-only reflectarray has easy fabrication, high antenna gain, high efficiency, and wide bandwidth. The concept of a metal-only antenna is widely used in [14], [15]. A novel approach based on superposition of overlapping rectangular metallic gratings was proposed to obtain scattering solutions of multiple gratings and design a fan-beam reflectarray [13]. The metal-only reflectarray illustrated in Fig. 2 is made of a feed and parallel-plate arrays. The reflectarray in Fig. 2 may be approximately modelled with multiple rectangular gratings [13].

In this work, we propose scattering solutions of multiple rectangular metallic gratings in a perfectly conducting plane excited by the TE and TM delta sources to maximize the antenna gain of a two-dimensional (2D) metal-only antenna. In order to analyze the overall characteristics of the metal-only antenna, we use the same method presented in [13]. Using an overlapping T-block method [13], we will deduce the simultaneous equations for the TE- and TM-modes. Solving these equations yields the radiation patterns and antenna directivity of a 2D metal-only antenna with a delta source.

\section{SIMULATION ALGORITHM}

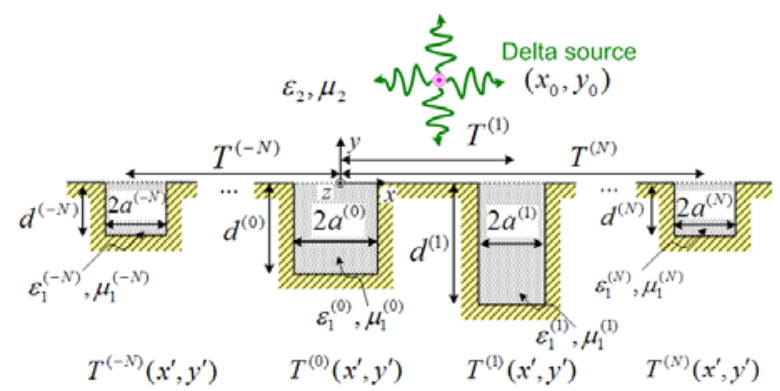

Fig. 3. Geometry of multiple rectangular metallic gratings

Consider the multiple rectangular gratings in a perfectly conducting plane shown in Fig. 3 illuminated by the TE $\left(E_{z}\right)$ and TM $\left(H_{z}\right)$ delta sources. In terms of the Hankel function, the incident and reflected $H_{z}$ and $E_{z}$ fields within a source block are represented as, respectively,

$$
\begin{aligned}
& S_{H}(x, y)=\frac{i}{4}\left\{H_{0}^{(1)}\left[k_{2} R_{x y}\left(x_{0}, y_{0}\right)\right]\right. \\
& \left.+H_{0}^{(1)}\left[k_{2} R_{x y}\left(x_{0},-y_{0}\right)\right]\right\} \\
& S_{E}(x, y)=\frac{i}{4}\left\{H_{0}^{(1)}\left[k_{2} R_{x y}\left(x_{0}, y_{0}\right)\right]\right. \\
& \left.-H_{0}^{(1)}\left[k_{2} R_{x y}\left(x_{0},-y_{0}\right)\right]\right\},
\end{aligned}
$$

where $\quad R_{x y}\left(x^{\prime}, y^{\prime}\right)=\sqrt{\left(x-x^{\prime}\right)^{2}+\left(y-y^{\prime}\right)^{2}} \quad$, $k_{2}=\omega \sqrt{\mu_{2} \varepsilon_{2}}$, and $H_{0}^{(1)}(\cdot)$ is the zeroth order Hankel function of the first kind.
Based on a standard mode-matching procedure, the $H_{z}$ and $E_{z}$ fields within a T-block $(-d<y<0)$ can be formulated as

$$
\begin{aligned}
& H_{z}(x, y)=\sum_{m=0}^{\infty} q_{m} \cos a_{m}(x+a) \cos \xi_{m}(y+d) \\
& E_{z}(x, y)=\sum_{m=1}^{\infty} p_{m} \sin a_{m}(x+a) \sin \xi_{m}(y+d)
\end{aligned}
$$

where $q_{m}$ and $p_{m}$ are unknown modal coefficients for the TM- and TE-modes with respect to discontinuity at $y=0$, respectively, $d$ and $2 a$ are depth and width of a rectangular groove in Fig. 3, respectively, $a_{m}=m \pi /(2 a)$, $\xi_{m}=\sqrt{k_{1}^{2}-a_{m}^{2}}$, and $k_{1}=\omega \sqrt{\mu_{1} \varepsilon_{1}}$.

\subsection{TM-Mode Analysis}

For the TM-mode analysis, the $H_{z}$ and $E_{x}$ fields continuities at $y=0$ should be enforced. In terms of the overlapping T-block method in [13], the $E_{x}$ field continuity at $y=0$ is automatically satisfied due to virtual current cancellation based on the Green's function relation. Multiplying the $H_{z}$ field continuity at $y=0$ by $\cos a_{l}^{(p)}\left(x-T^{(p)}+a^{(p)}\right) \quad(\quad l=0,1, \cdots \quad$ and $p=-N,-N+1, \cdots, N-1, N$ ) and integrating over $T^{(p)}-a^{(p)}<x<T^{(p)}+a^{(p)} \quad$ yields the scattering solutions for the TM-mode as

$$
\sum_{n=-N}^{N} \sum_{m=0}^{\infty} q_{m}^{(n)} I_{H}^{(n)}\left(T^{(p)}-T^{(n)} ; a^{(p)}\right)=s_{H, l}^{(p)},
$$

where the total number of grooves is $2 N+1, q_{m}^{(n)}$ is the $m$ th unknown TM modal coefficient of the $n$th T-block $T^{(n)}\left(x^{\prime}, y^{\prime}\right)$ in Fig. 3, $(\cdot)^{(p)}$ is a geometric parameter for the $p$ th T-block, $a_{l}^{(p)}=l \pi /\left(2 a^{(p)}\right)$,

$$
\begin{aligned}
& I_{H}^{(n)}\left(x_{0} ; a^{(p)}\right)=\cos \left(\xi_{m}^{(n)} d^{(n)}\right) a^{(n)} \alpha_{m} \delta_{m l}^{n p} \\
& +\frac{\varepsilon_{2}}{\varepsilon_{1}^{(n)}} \xi_{m}^{(n)} \sin \left(\xi_{m}^{(n)} d^{(n)}\right) \\
& \times\left[\frac{a^{(n)} \alpha_{m} \delta_{m l}^{n p}}{i \eta_{m}^{(n)}}+I_{m l}^{H}\left(x_{0}\right)\right]
\end{aligned}
$$


$S_{H, l}^{(p)}=\frac{i}{2} \int_{T^{(p)}{ }_{-a}^{(p)}}^{T^{(p)}+a_{0}^{(p)}} H_{0}^{(1)}\left[k_{2} R_{x 0}\left(x_{0}, y_{0}\right)\right]$

$\cdot \cos a_{l}^{(p)}\left(x-T^{(p)}+a^{(p)}\right) d x$

$I_{m l}^{H}\left(x_{0}\right)=\frac{k_{2}}{\pi} \int_{0}^{\infty} \frac{(1+2 v i) \xi}{\left(\xi^{2}-a_{m}^{2}\right)\left(\xi^{2}-c_{l}^{2}\right)}$

$\left.\cdot f_{m l}^{H}\left(x_{0} ; \xi\right) d v\right|_{\xi=k_{2} \sqrt{1-v^{2}(v-i)^{2}}} ^{a=a^{(n)}, c=a(p)}$

$f_{m l}^{H}\left(x_{0} ; \xi\right)=(-1)^{m} e^{i \xi\left|x_{0}-a-c\right|}+(-1)^{l} e^{i \xi\left|x_{0}+a+c\right|}$

$-(-1)^{m+l} e^{i \xi\left|x_{0}-a+c\right|}-e^{i \xi\left|x_{0}+a-c\right|}$,

$k_{1}^{(n)}=\omega \sqrt{\mu_{1}^{(n)} \varepsilon_{1}^{(n)}} \quad, \quad \xi_{m}^{(n)}=\sqrt{\left(k_{1}^{(n)}\right)^{2}-\left(a_{m}^{(n)}\right)^{2}}$,

$\eta_{m}^{(n)}=\sqrt{k_{2}^{2}-\left(a_{m}^{(n)}\right)^{2}} \quad, \quad \alpha_{m}=\delta_{m 0}+1$,

$\delta_{m l}^{n p}=\delta_{m l} \delta_{n p}$, and $\delta_{m l}$ is the Kronecker delta.

\subsection{TE-Mode Analysis}

Similar to the TM-mode analysis, we utilize the overlapping T-block approach to obtain the scattering analysis of the TEmode. By multiplying the $H_{x}$ continuity at $y=0$ with $\sin a_{l}^{(p)}\left(x-T^{(p)}+a^{(p)}\right) \quad(\quad l=1,2, \cdots \quad$ and $p=-N,-N+1, \cdots, N-1, N$ ) and integrating with respect to $X$, we get

$$
\sum_{n=-N}^{N} \sum_{m=1}^{\infty} p_{m}^{(n)} I_{E}^{(n)}\left(T^{(p)}-T^{(n)} ; a^{(p)}\right)=s_{E, l}^{(p)},
$$

where $p_{m}^{(n)}$ is the $m$ th unknown TE modal coefficient for the $n$th T-block,

$$
\begin{aligned}
& I_{E}^{(n)}\left(x_{0} ; a^{(p)}\right)=\frac{1}{\mu_{1}^{(n)}} \xi_{m}^{(n)} \cos \left(\xi_{m}^{(n)} d^{(n)}\right) a^{(n)} \delta_{m l}^{n p} \\
& -\frac{1}{\mu_{2}} \sin \left(\xi_{m}^{(n)} d^{(n)}\right) \\
& \times\left[i \eta_{m}^{(n)} a^{(n)} \delta_{m l}^{n p}+I_{m l}^{E}\left(x_{0}\right)\right] \\
& S_{E, l}^{(p)}=\frac{i k_{2} y_{0}}{2 \mu_{2}} \int_{T^{(p)}}^{T^{(p)}(p)} \frac{H_{1}^{(1)}\left[k_{2} R_{x 0}\left(x_{0}, y_{0}\right)\right]}{R_{x 0}\left(x_{0}, y_{0}\right)} \\
& \cdot \sin a_{l}^{(p)}\left(x-T^{(p)}+a^{(p)}\right) d x \\
& I_{m l}^{E}\left(x_{0}\right)=-\frac{k_{2} a_{m} C_{l}}{\pi} \int_{0}^{\infty} \frac{(1+2 v i) \eta^{2}}{\xi\left(\xi^{2}-a_{m}^{2}\right)\left(\xi^{2}-c_{l}^{2}\right)} \\
& \left.\cdot f_{m l}^{H}\left(x_{0} ; \xi\right) d v\right|_{\xi=k_{2} \sqrt{1-v^{2}(v-i)^{2}}} ^{a={ }^{(n)}},
\end{aligned}
$$

and $\eta=k_{2} v(v-i)$.

\section{NUMERICAL COMPUTATIONS}

To verify our approach, we check the $H_{z}$ and $H_{x}$ field continuities at $y=0$ for the TM and TE delta source excitations, respectively. As the truncated number of modes in region (I) in (5) and (10) increases, the $H_{z}$ and $H_{x}$ fields between regions (I) $(y<0)$ and (II) ( $y>0$ ) become more continuous, thus confirming that our solutions in (5) and (10) are well-behaved.

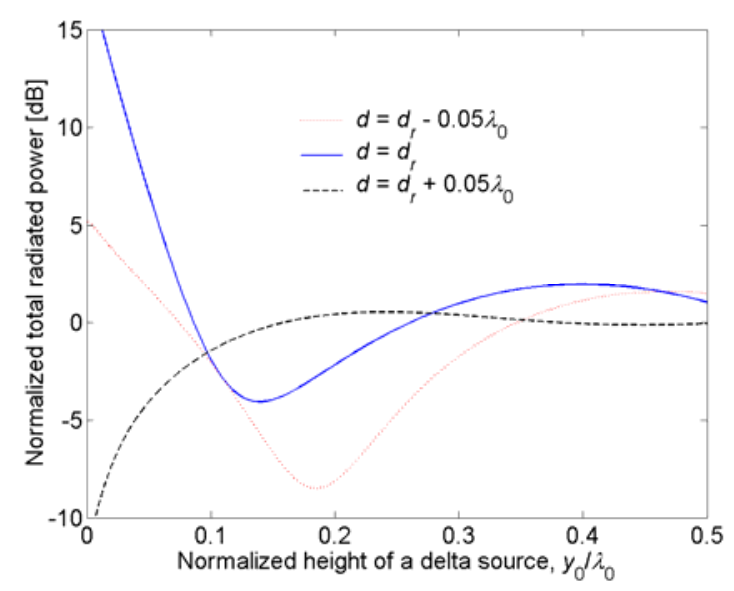

(a) Total radiated power

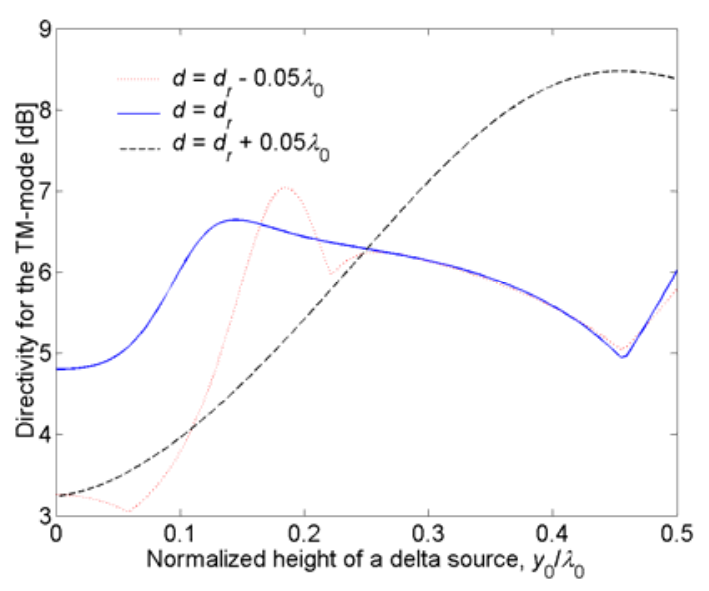

(b) Antenna directivity

Fig. 4. Behaviors of total radiated power and antenna directivity versus the position of the TM delta source $y_{0}$ with

$$
\begin{gathered}
N=1, M=4, a=0.025 \lambda_{0}, T=0.1 \lambda_{0}, \\
x_{0}=0, \varepsilon_{1}^{(n)}=\varepsilon_{2}=\varepsilon_{0}, \text { and } \mu_{1}^{(n)}=\mu_{2}=\mu_{0}
\end{gathered}
$$

The geometric dimensions of multiple rectangular gratings in Fig. 3 are chosen to show superdirective radiation proposed in [16]. In this computation, we assume that $a^{(n)}=a$, 
$T^{(n)}=n T$, the total number of grooves $2 N+1=3$, the number of modes $M=4$ for simplicity. For the TM planewave incidence, the cavity resonance formed by a rectangular groove or the $\Pi$-mode [16] is found with the variation of $d$, when $a=0.025 \lambda_{0}$, and $T=0.1 \lambda_{0}$. Note that $\lambda_{0}$ denotes a free-space wavelength. This procedure to find $d$ has been verified in [16]. The determined value for $d$ is $d_{r}=0.219927 \lambda_{0}$. However, the plane-wave incidence [16] is not realistic in practical applications. In order to find the feasibility of superdirective radiation, we need to use a delta source excitation.

Fig. 4 shows the behaviors of total radiated power and antenna directivity versus the position of the TM delta source $y_{0}$. The scattered fields from multiple rectangular grooves are normalized with $\frac{i}{4} H_{0}^{(1)}\left[k_{2} R_{x y}(0,0)\right]$. When $d=d_{r}$ and $y_{0}$ goes to zero, the total radiated power for the TM delta source in Fig. 4(a) becomes very large. These characteristics indicate that superdirective radiation for a planewave incidence results in large radiation power for a delta source excitation. In addition, when $d$ deviates from $d_{r}$, the total radiated power becomes smaller. However, in Fig. 4(b), we compute the antenna directivity of multiple rectangular grooves with superdirective radiation. In this case, the directivity is less than $9[\mathrm{~dB}]$, thus confirming that the concept proposed in [16] is not applied to the delta source excitation.

For the TE plane-wave incidence, the cavity resonance or the $\Pi$-mode is not observed in our numerical computations and total radiated power and directivity for the TE delta source are kept in normal value. This is because the groove width $2 a$ is very small compared to the wavelength and thus the PEC with multiple corrugated grooves almost acts as a plain PEC plane.

Table 1. Calculated $d^{(n)}[\mathrm{mm}]$ for the TM delta source

\begin{tabular}{|c|c|c|c|c|c|}
\hline$n$ & $d^{(n)}$ & $n$ & $d^{(n)}$ & $n$ & $d^{(n)}$ \\
\hline 0 & 0.007 & 10 & 1.394 & 20 & 0.559 \\
\hline 1 & 2.494 & 11 & 1.161 & 21 & 0.103 \\
\hline 2 & 2.461 & 12 & 0.905 & 22 & 2.124 \\
\hline 3 & 2.405 & 13 & 0.627 & 23 & 1.624 \\
\hline 4 & 2.327 & 14 & 0.327 & 24 & 1.102 \\
\hline 5 & 2.227 & 15 & 0.005 & 25 & 0.557 \\
\hline 6 & 2.105 & 16 & 2.159 & 26 & 2.489 \\
\hline 7 & 1.961 & 17 & 1.792 & 27 & 1.900 \\
\hline 8 & 1.794 & 18 & 1.403 & 28 & 1.289 \\
\hline 9 & 1.605 & 19 & 0.992 & 29 & 0.656 \\
\hline
\end{tabular}

In order to design a high directivity antenna, we use the variation of $d$ as [13] $d^{(n)}=d_{0}\left[1-\left(\frac{2 T^{(n)}}{T_{0}}\right)^{2}\right] \frac{\lambda_{g}}{\lambda_{0}}$

where

$$
T^{(n)}=n T
$$

$T_{0}=16 d_{0} \cdot \mathrm{RA} \approx T^{(-N)}+T^{(N)}+a^{(-N)}+a^{(N)}$

RA ( $\left.=f_{0} / T_{0}\right)$ is a relative aperture of multiple rectangular gratings, $f_{0}$ is a focus, $d_{0}$ is the maximum depth of grooves, and $\lambda_{g}$ is a guided wavelength within a rectangular groove. Note that (14) is obtained based on the phase of a parabolic reflector [13].

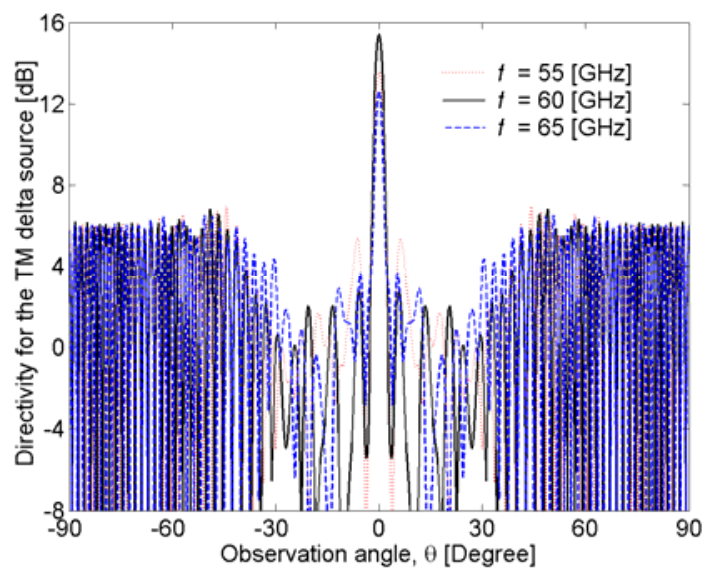

Fig. 5. Calculations of the TM-mode directivity versus an observation angle $\theta$ with $N=29, M=3$,

$$
a=0.5[\mathrm{~mm}], T=2[\mathrm{~mm}], x_{0}=0 \text {, }
$$

$y_{0}=80[\mathrm{~mm}], \varepsilon_{1}^{(n)}=\varepsilon_{2}=\varepsilon_{0}, \mu_{1}^{(n)}=\mu_{2}=\mu_{0}$,

$\mathrm{RA}=0.75, d_{0}=10[\mathrm{~mm}], T_{0}=120[\mathrm{~mm}]$, and

$$
d^{(n)} \text { defined in Table } 1
$$

Fig. 5 illustrates the behaviors of antenna directivity versus an observation angle $\theta$ for the TM delta source excitation, where $\theta=\tan ^{-1}(x / y)$. For $f=60 \quad[\mathrm{GHz}]$, the parameter $d^{(n)}$ is calculated with (14) and shown in Table 1. When $d^{(n)}>\lambda_{g} / 2, d^{(n)}$ can be less than $\lambda_{g} / 2$ by using the periodicity of a reflection coefficient. The $y_{0}$ position of a delta source is determined with $y_{0}=f_{0}-d_{0} \lambda_{g} / \lambda_{0}$. In Fig. 5 , the reflected main-beam for the TM delta source is at $\theta=0^{\circ}$ and the directivities for 55, $60,65[\mathrm{GHz}]$ are $13.7,15.6,13[\mathrm{~dB}]$, respectively. This indicates that our design formula (14) works very well for a high directivity antenna. 


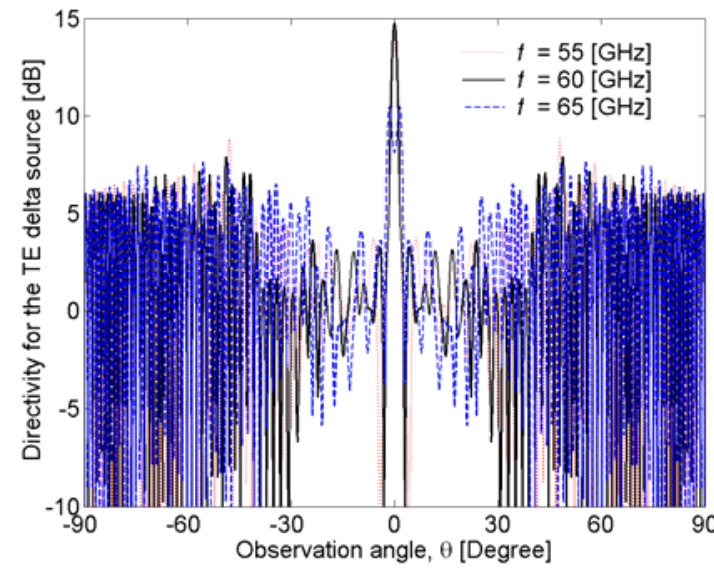

Fig. 6. Calculations of the TE-mode directivity versus an observation angle $\theta$ with $N=19, M=3$,

$$
\begin{gathered}
a=2[\mathrm{~mm}], T=4.5[\mathrm{~mm}], x_{0}=0, \\
y_{0}=115.793[\mathrm{~mm}], \varepsilon_{1}^{(n)}=\varepsilon_{2}=\varepsilon_{0}, \\
\mu_{1}^{(n)}=\mu_{2}=\mu_{0}, \mathrm{RA}=0.75, d_{0}=15[\mathrm{~mm}], \\
T_{0}=180[\mathrm{~mm}], \text { and } d^{(n)} \text { defined in Table } 2
\end{gathered}
$$

Table 2. Calculated $d^{(n)}[\mathrm{mm}]$ for the TE delta source

\begin{tabular}{|r|c|c|c|c|c|c|c|}
\hline \multicolumn{1}{|c|}{$n$} & $d^{(n)}$ & $n$ & $d^{(n)}$ & $n$ & $d^{(n)}$ & $n$ & $d^{(n)}$ \\
\hline 0 & 0.013 & 5 & 2.012 & 10 & 1.609 & 15 & 2.005 \\
\hline 1 & 3.164 & 6 & 1.484 & 11 & 0.601 & 16 & 0.517 \\
\hline 2 & 3.020 & 7 & 0.859 & 12 & 2.696 & 17 & 2.131 \\
\hline 3 & 2.780 & 8 & 0.139 & 13 & 1.495 & 18 & 0.450 \\
\hline 4 & 2.444 & 9 & 2.522 & 14 & 0.199 & 19 & 1.873 \\
\hline
\end{tabular}

Similar to Fig. 5, Fig. 6 illustrates the behaviors of the TEmode directivity. The simulation was performed based on Table 2. The calculated directivities for 55, 60, 65 [GHz] are obtained as 14, 15, 10.5 [dB], respectively.

\section{CONCLUSION}

The TE and TM scattering equations to obtain high antenna directivity were proposed for multiple rectangular metallic gratings in a perfectly conducting plane excited by delta sources. Utilizing a standard mode-matching technique combined with an overlapping T-block method, we obtained the simultaneous equations for the TE- and TM-modes. To obtain high directivity, we changed the depth of grooves in terms of superdirective radiation and parabolic reflector phase. Our computations showed that superdirective radiation is not suitable for the delta source. However, the depth variation based on parabolic reflector phase produced very high directivity with fan-beam radiation.

Using the design algorithm for a delta source and the phase center information of a feed, we can design very high directivity fan-beam reflectarray with an arbitrary feed.
In the future work, we will fabricate a metal-only fan-beam reflectarray for an electromagnetic fence. To accomplish this, we will need to design a two-dimensional feed which radiates the two-dimensional TE- or TM-modes.

\section{ACKNOWLEDGEMENT}

This study was financially supported by academic research fund of Mokwon University in 2011.

\section{REFERENCES}

[1] P. Blacksmith, L.J. Poirier, F.S. Holt, "Radar intrusion detection system,” U.S. Patent No. 4132988, Jan. 1979.

[2] J. Bjornholt, G. Hamman, S. Miller, "Electronic fence using high-resolution millimeter-wave radar in conjunction with multiple passive reflectors," U.S. Patent No. 6466157, Oct. 2002.

[3] D.M. Anderson "Virtual fencing-past, present and future,” The Rangeland Journal, vol. 29, no. 1, 2007, pp. 65-78.

[4] M.O. Monod, P. Faure, L. Moiroux, P. Rameau, "A virtual fence for animals management in rangelands," The 14th IEEE Mediterranean Electrotechnical Conference, May 2008, pp.337-342.

[5] A.O. Salman, D. Dibekci, S.P. Gavrilov, and A.A. Vertiy, "The radiation properties of a novel wire antenna for the security fence radar,” IEEE Trans. Antennas Propagat., vol. 56, no. 9, Sept. 2008, pp. 2852-2864.

[6] I. Ituen and G.-H. Sohn, "The environmental applications of wireless sensor networks," International Journal of Contents, vol. 3, no. 4, Dec. 2007, pp. 1-7.

[7] S.-J. Kim, M.-S. Hwang, and Y.-M. Kim, "Implementation of low power algorithm for near distance wireless communication and RFID/USN systems," International Journal of Contents, vol. 7, no. 1, March 2011, pp. 1-7.

[8] A. Zeitler, J. Lanteri, C. Pichot, C. Migliaccio, P. Feil, and W. Menzel, "Folded reflectarrays with shaped beam pattern for foreign object debris detection on runways," IEEE Trans. Antennas Propagat., vol. 58, no. 9, Sept. 2010, pp. 3065-3068.

[9] Z. Pi and F. Khan, "An introduction to millimeter-wave mobile broadband systems," IEEE Communications Magazine, vol. 49, no. 6, June 2011, pp.101-107.

[10] D.G. Berry, R.G. Malech, and W.A. Kennedy, "The reflectarray antenna,” IEEE Trans. Antennas Propagat., vol. 11, no. 6, Nov. 1963, pp. 645-651.

[11] J. Huang and J.A. Encinar, Reflectarray Antennas. Hoboken-Piscataway: Wiley-IEEE Press, 2007.

[12] A.G. Roederer, "Reflectarray antennas," European Conference on Antennas and Propagation, pp. 18-22, March 2009.

[13] Y.H. Cho, W.J. Byun, and M.S. Song, "Metallicrectangular-grooves based 2D reflectarray antenna excited by an open-ended parallel-plate waveguide,” 
IEEE Trans. Antennas Propagat., vol. 58, no. 5, May 2010, pp. 1788-1792.

[14] M. Ando, K. Sakurai, N. Goto, K. Arimura, and Y. Ito, "A radial line slot antenna for $12 \mathrm{GHz}$ satellite TV reception,” IEEE Trans. Antennas Propagat., vol. 33, no. 12, Dec. 1985, pp. 1347- 1353.

[15] A. Lemons, R. Lewis, W. Milroy, R. Robertson, S. Coppedge, and T. Kastle, "W-band CTS planar array," IEEE Microwave Symposium Digest, vol. 2, 1999, pp. 651-654.

[16] D.C. Skigin, V.V. Veremey, and R. Mittra, "Superdirective radiation from finite gratings of rectangular grooves,” IEEE Trans. Antennas Propagat., vol. 47, no. 2, Feb. 1999, pp. 376-383.

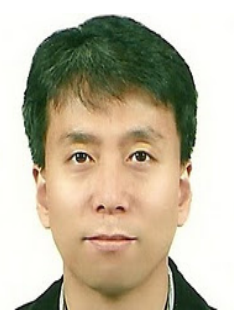

\section{Yong-Heui Cho}

He received the $B$. S. degree in Electronics Engineering from the Kyungpook National University, Daegu, Korea, in 1998, the M. S. and Ph. D. degrees in Electrical Engineering from the Korea Advanced Institute of Science and Technology (KAIST), Daejeon, Korea, in 2000 and 2002, respectively. From 2002 to 2003, he was a Senior Research Staff with the Electronics and Telecommunications Research Institute (ETRI), Daejeon, Korea. In 2003, he joined the School of Information and Communication Engineering, Mokwon University, Daejeon, Korea, where he is currently an Associate Professor. In 2011, he is on the sabbatical leave with the Department of Electrical and Computer Engineering, University of Massachusetts Amherst, MA, USA. His research interests include dispersion characteristics of waveguides, electromagnetic wave scattering, and design of reflectarrays. 